\title{
Determination of Tensile Properties of Polymers at High Strain Rates
}

\author{
M. Reiter ${ }^{1}$ and Z. Major ${ }^{1}$ \\ ${ }^{1}$ Johannes Kepler University, Institute of Polymer Product Engineering, Linz, Austria
}

\begin{abstract}
In the field of high rate testing of polymers the measured properties are highly dependent on the applied methodology. Hence, the test setup as whole but in particular also the geometrical type of specimen plays a decisive role. The widely used standard for the determination of tensile properties of polymers (ISO527-2) was extended by a novel standard (ISO18872:2007), which is targeted on the determination of tensile properties at high strain rates. In this standard also a novel specimen shape is proposed. Hand in hand with the introduction of new specimen geometry the question of comparability arises. To point out the differences in stress-strain response of the ISO18872 specimen and the ISO527-2 multipurpose specimen tensile tests over a wide loading rate range were conducted in this paper. A digital image correlation system in combination with a high speed camera was used to characterize the local material behaviour. Different parameters like nominal stress, true stress, nominal strain, true strain as well as volumetric strain were determined and used to compare the two specimen geometries.
\end{abstract}

\section{Introduction}

Since the appliance of FEM-simulations for predicting component behaviour is getting more and more important, the demand for reliable material models and material parameters for these simulations is rising as well. Especially in the automotive industry reliable material parameter for high loading rates and high strains are required. Besides various problems involved in impact testing (like data acquisition, system oscillations, etc.) the question of the right specimen geometry remains always very essential. In the polymer industry the widely used standard for the determination of tensile properties is the ISO527-2 [1], which provides the definition for the multipurpose specimen. This standard aims to tests at low to moderate loading rates. For material testing at high loading rates a novel standard (ISO18872:2007) was introduced [2]. Along with it also novel specimen geometry was defined.

In this paper the two specimen geometries from these standards are compared in detail. Therefore, a polypropylene material was chosen and uniaxial tensile tests at low and high loading rates were conducted. For adequate material modelling and FE-simulation of polymeric materials, proper true stress - true strain data are necessary. Thus, a digital image correlation system [3, 4] in combination with a high speed camera was used to characterize the local material behaviour. Different measures like nominal stress, true stress, nominal strain, true strain as well as volumetric strain were determined over a wide loading rate range and used to compare the two different specimen geometries investigated.

This is an Open Access article distributed under the terms of the Creative Commons Attribution-Noncommercial License 3.0, which permits unrestricted use, distribution, and reproduction in any noncommercial medium, provided the original work is properly cited. 


\section{Experimental}

Tensile tests were conducted at slow loading rate of $0.1 \mathrm{~mm} / \mathrm{s}$ as well as high loading rates of $500 \mathrm{~mm} / \mathrm{s}$ and $8000 \mathrm{~mm} / \mathrm{s}$ at room temperature $\left(23^{\circ} \mathrm{C}\right)$ in this study.

\subsection{Material and specimens}

Due to its stable and well-characterized deformation and fracture behaviour, for the comparison of the two specimen types a polypropylene $(\mathrm{PP}(\mathrm{H}))$ Borealis, Linz, A) material was investigated.

The ISO 18872:2007 proposes novel specimen geometry for the determination of tensile properties under impact loading. The specimen shape is similar to the multipurpose specimen defined by ISO527-2. The main difference is an additional reduction of the effective cross-section in the middle of the specimen with a nominal radius of $35 \mathrm{~mm}$ as shown in figure 1 .

For the experiments presented in this paper the ISO18872 specimens were machined from injection moulded ISO 527 multipurpose specimens.

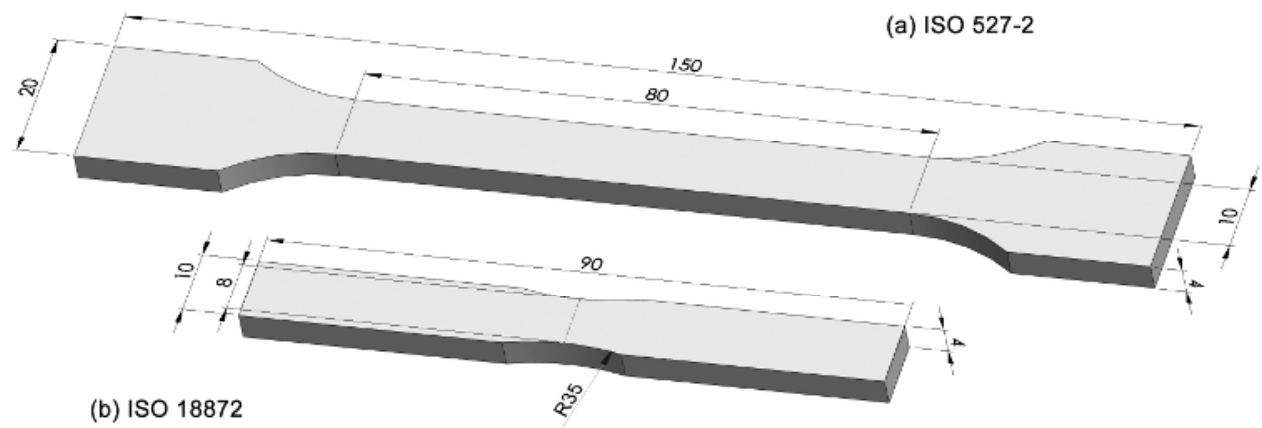

Fig. 1 Specimen Geometry a) ISO527-2, b) ISO 18872

\subsection{Setup of the uniaxial tensile test}

All tensile tests were performed using a high rate servohydraulic testing machine (Polymer Test System, MTS831, MTS Systems GmbH, Berlin, D), which provides a high flexibility in varying the loading rate from 0.001 up to $8000 \mathrm{~mm} / \mathrm{s}$. The slow tests at $0.1 \mathrm{~mm} / \mathrm{s}$ loading rate were performed using a low rate setup. For the high rate setup an additional slack adapter [5] was used, which solves the problem that one specimen grip has to be accelerated instantaneously to the nominal testing speed. This slack adapter allows the hydraulic cylinder to accelerate to the desired speed before striking the specimen off.

\subsubsection{Force measurement}

In the low rate setup the force was measured by a common strain gauge load cell of the testing machine. Since this load cell is not optimized for dynamic processes a piezoelectric longitudinal measuring pin (Kistler 9243A, Winterthur, $\mathrm{CH}$ ) integrated in the lower specimen grip was used to measure the force for the high rate tests. The direct integration of the piezoelectric sensor into the specimen grip and a lightweight construction leads to a high resonance frequency which is absolutely necessary for accurate force measurements at these loading rates. 


\subsubsection{Strain measurement - digital image correlation}

In principle the strain of the specimen can be measured globally or locally. Using a global consideration the nominal strain can be obtained through measurements of the change in the grip separation. That was measured by the linear displacement velocity transducer signal (LVDT) of the test system. Using this method as basis for strain calculation it is not possible to detect any strain localization effects. The use of a slack adapter invokes an additional system compliance [6], which cannot be separated from the specimen compliance and therefore generates unreliable results. In this case the strain should be measured locally.

To comply with this requirement, a full-field strain analysis was applied using a digital image correlation system (Aramis by GOM, Braunschweig, D). Therefore the specimens were sprayed with two lack system resulted in a stochastic black and white pattern on the specimen surface. By recording this pattern during the test with a video camera system it is possible to calculate the strain field for each frame.

In order to record images over the wide loading rate range from $0.1 \mathrm{~mm} / \mathrm{s}$ up to $8000 \mathrm{~mm} / \mathrm{s}$ it was necessary to use two different camera systems. For the low rate tests two medium speed cameras (Basler, $\mathrm{CH}$ ) with a maximum frame rate of 8000 frames/s were applied. Whereas a high speed camera (Photron SA1, UK) with a recording speed up to 675.000 frames/s was used for the high rate tests.

To calculate the local strain field the image is divided into subsets with a size of $0.6 \mathrm{~mm}$ to $0.7 \mathrm{~mm}$. The subset size is limited by the pixel resolution of the camera. By identifying the position and the deformation of these subsets (or facets) in every frame the strain field can be obtained. The geometrical resolution of this strain field corresponds to the size of the facets.

Regarding the global strain measurement, where the gauge length equals the distance between the grips, all effects of non-uniform strain distribution within this gauge length cannot be detected. In comparison, the local strain measurement gives strain information on multiple locations with a gauge length of the facet size. This measuring method brings the advantage that one can take deeper look at the true material behaviour.

Because the true stress - true strain behaviour is one of the fundamental material properties and is absolutely necessary for reliable material modelling, the local material behaviour has to be taken into account. As one can see in figure 2 the strain distribution is non-uniform for both specimen geometries. Therefore the cross-section with the highest strain concentration was determined first. All following evaluation steps were based on the facets in this cross-section only.
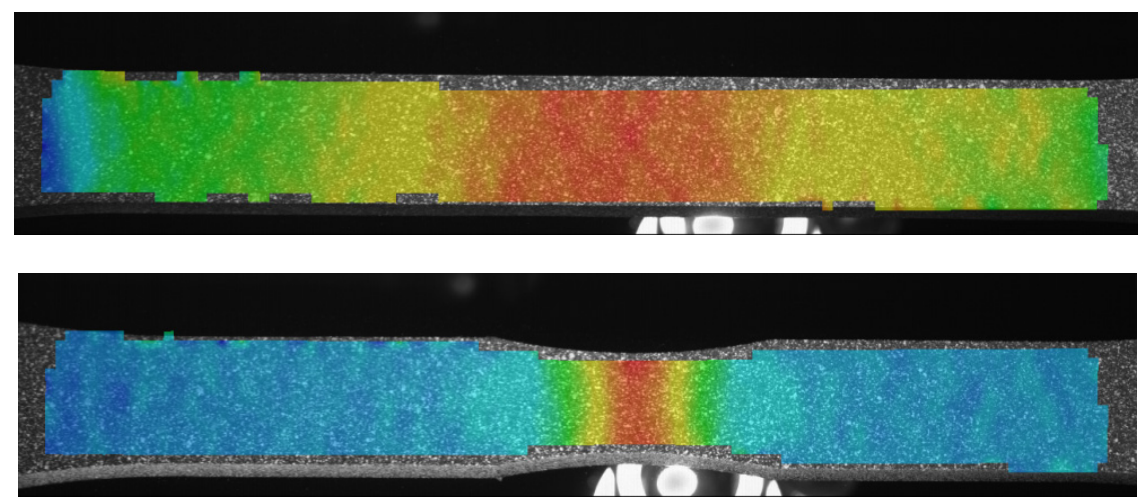

Fig. 2. Full-field strain images of uniform (ISO 527) and smooth notched (ISO 18872) specimens at the same displacement level. 


\subsection{Data reduction}

Since the digital image correlation system provides not only the longitudinal but also the transversal strains, the actual cross-section can be determined at any time. With this additional information the true stress, the Poisson's ratio as well as the volumetric strain can be calculated.

\subsubsection{Local strain, global strain}

The nominal global strain is calculated by the measurement of the displacement of the hydraulic cylinder $s$ and the specimen gauge length $L_{0}$ as

$$
\varepsilon_{G}^{N}=\frac{s}{L_{0}}
$$

Regarding the local deformations a nominal as well as a true strain can be defined. The nominal strain $\varepsilon^{\mathrm{N}}$ is always relating to the initial dimensions of the specific facet. The true strain $\varepsilon$ (also called logarithmic or natural strain) is defined as the logarithm of the stretch ratio $\lambda$.

$$
\varepsilon=\ln (\lambda) \text { with } \lambda=1+\varepsilon
$$

These strain values are provided by the image correlation system as an average value of all facets in the relevant cross section.

\subsubsection{True stress}

The true stress, $\sigma_{t}$ considers the reduction of the cross section during tensile loading. For the calculation of the actual cross section both transversal strains should be known. Since the front side of the specimen was the only side that was observed, only one transversal strain $\varepsilon_{\text {trans }}$ was available. Thus, the other transversal strain was assumed to be equal to $\varepsilon_{\text {trans }}$ [3]. That leads to the definition of $\sigma_{\mathrm{t}}$ as

$$
\sigma_{t}=\frac{F}{A_{0}\left(1+\varepsilon_{\text {trans }}\right)^{2}}
$$

\subsubsection{Volumetric strain}

During uniaxial tensile test several polymeric materials shows a volume increase. This volume change is effected by different mechanisms like elastic dilatation, void and craze formation. For a better understanding of these mechanisms the volume strain has to be measured. Since the longitudinal and transversal strain ( $\varepsilon_{\text {long }}$ and $\varepsilon_{\text {trans }}$ ) is available, it is possible to determine the volume strain as well. The volume strain can also be expressed as nominal strain or as true (logarithmic) strain. In this paper the following definition for the nominal volume strain [7] is used.

$$
\varepsilon_{V}=\frac{\Delta V}{V_{0}}=\left(1+\varepsilon_{\text {long }}\right)\left(1+\varepsilon_{\text {trans }}\right)^{2}
$$

An isotropic material behavior was supposed, where both transversal strains are assumed to be equal. 


\section{Results and Discussion}

In order to point out the difference between a local and a global strain measurement the different stress and strain data were calculated for the high rate tensile test with $500 \mathrm{~mm} / \mathrm{s}$. As one can see in figure 3 the global nominal strain measurement produces inaccurate results compared to the local measurement. At the beginning the strains are overestimated due to the system compliance. This is mainly induced by the damping elements in the slack adapter, which are used to reduce the effect of oscillations caused by the impact pulse. As the strain localizes after reaching the yield point the strains are underestimated. For the impact specimen (ISO18872) the effect of strain localization occurs practically ab initio. Thus, for this type of specimen a local strain measurement is obligatory.

Taking the actual cross section into account, the true stress which is important for material modelling purpose, can be calculated. As one can see the true stress shows apparent hardening whereas the nominal strain suggests an apparent strain softening for this material.
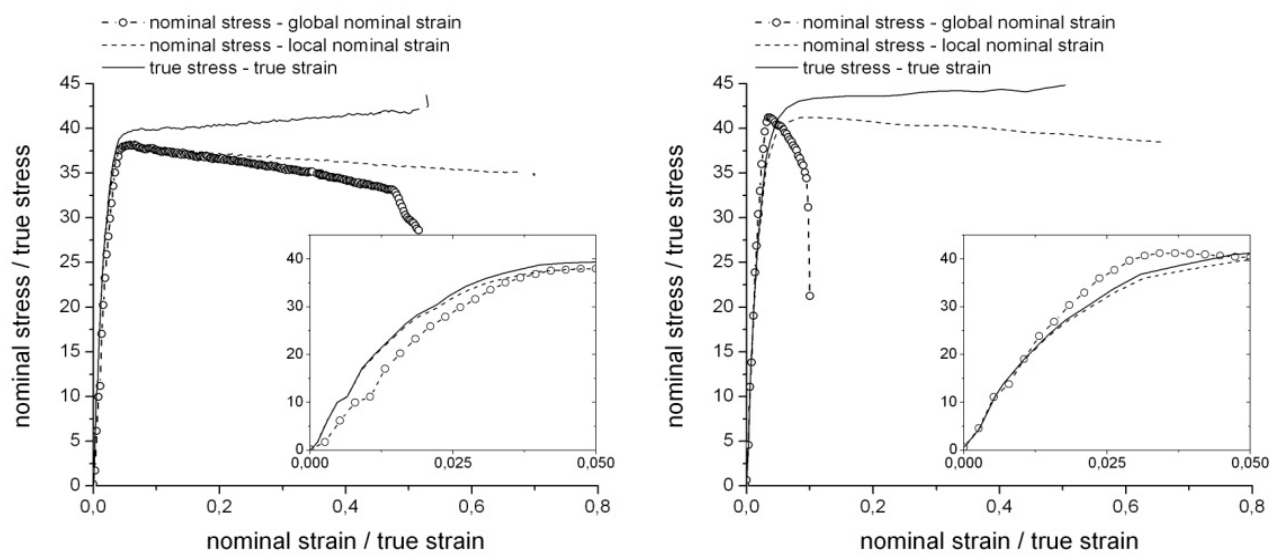

Fig. 3. Comparison of nominal stress - nominal strain and true stress - true strains;

(a) ISO527 specimen at $500 \mathrm{~mm} / \mathrm{s}$; (b) ISO18872 specimen at $500 \mathrm{~mm} / \mathrm{s}$

Another important parameter for the material characterization is the strain rate, which is defined as the rate of change in strain with respect to time. It can also be related to the nominal strain as well as the true strain. In figure 4 the difference between the strain rate derived from true strain and nominal strain is pointed out. In general the true strain rate is lower than the nominal strain rate. As one can see the two curves are relatively similar before reaching the yield point. Thereafter the strain rate calculated from nominal strain is significantly higher.

Another aspect in this figure is the totally different strain rate characteristic between the ISO527 and the ISO18872 specimen. The initial strain rate for both specimen types is about $5 \mathrm{~s}^{-1}$ as expected for this specimen size. For the specimen with reduced cross section the initial strain rate is slightly higher. At the beginning of the test the strain rate increases for both specimen types. Compared to the uniform multipurpose specimen, which reaches an increase in strain rate by a factor of 1.5 at a strain level near the yield point, the ISO18872 specimen shows at the same strain level an increase in strain rate of about one decade. This result corresponds very well to the visualized strain field (figure 1) which shows significant higher strain localization for the ISO18872 specimen. 


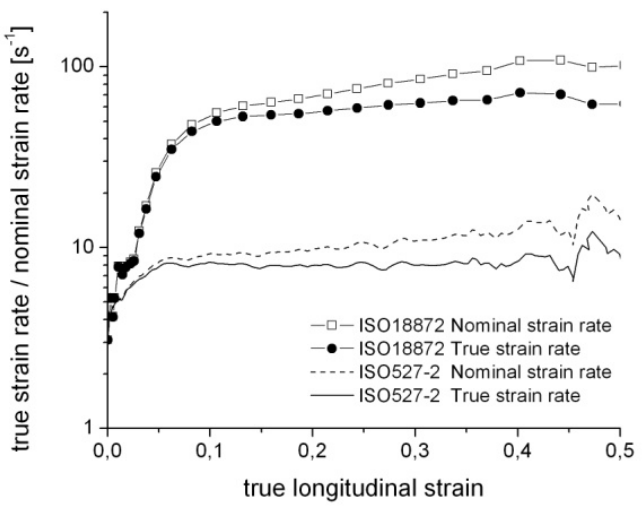

Fig. 4. Comparison of nominal strain rate and true strain rate with ISO527 and ISO18872 specimens at $500 \mathrm{~mm} / \mathrm{s}$

Figure 5 shows the different true stress - true strain response as well as the strain rate response for both specimen geometries and three testing rates. As mentioned before the ISO18872 specimen shows an increase in strain rate of approximately one decade in all testing rates. According to the higher strain rate the stress response is also higher. A very interesting point is that for the $500 \mathrm{~mm} / \mathrm{s}$ and $8000 \mathrm{~mm} / \mathrm{s}$ test a direct correlation between strain rate and stress response can be observed. Regarding the true stress of the ISO18872 specimen at $500 \mathrm{~mm} / \mathrm{s}$ one can see that at low strains the curve fits very well to the ISO527 specimen at the same $500 \mathrm{~mm} / \mathrm{s}$ loading rate. The strain rate increases till it reaches the strain rate level of the ISO527 specimen at the next higher loading rate $(8000 \mathrm{~mm} / \mathrm{s})$. As the strain rate increases the true stress also increases to that level of the ISO527 specimen at $8000 \mathrm{~mm} / \mathrm{s}$.
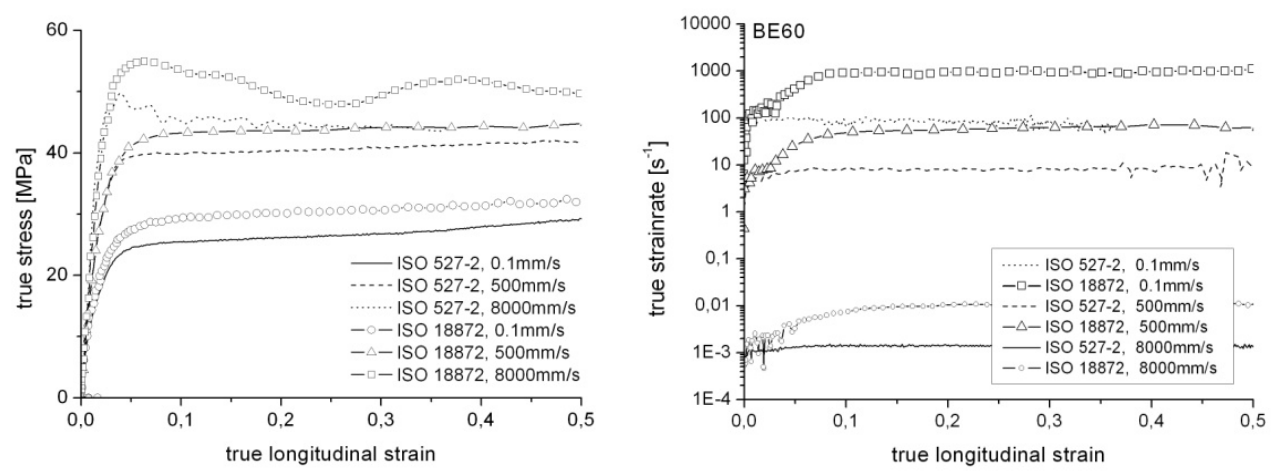

Fig. 5. (a) True stress - true strain; (b) true strain rate - true strain

This polypropylene material has an extensive volume strain in the post-yield deformation regime as one can see in figure 6, where the nominal volume strain is plotted over the true longitudinal strain. The amount of volume strain depends on the loading rate and the type of specimen, the higher the loading rate the higher the volume strain. 


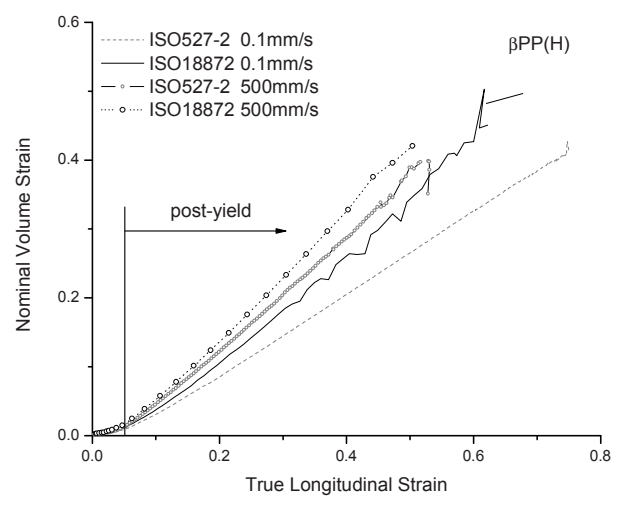

Fig. 6. Nominal volume strain - true strain

\section{Conclusion}

When comparing the ISO18872 high rate tensile specimen geometry and the ISO527 multipurpose specimen geometry regarding uniaxial tensile tests many differences in the stress-strain response can be shown. These differences can either be seen as advantage or disadvantage depending on the application.

The multipurpose specimen (ISO527) shows a relatively constant true strain rate over a large deformation range, which is important for the material modelling where true stress vs. true strain at constant strain rates are often required. The region where strain localization or necking takes place is previously not defined and depends on the many factors (i.e., accuracy of the shape, distribution of imperfections and inertia effects).

In contrary, the high rate tensile specimen (ISO18872) reveals a non-uniform strain rate profile, the strain rate increases during the test by approximately one decade. At low strains the strain rate is similar to the ISO527 specimen. The smooth neck in the middle of the specimen acts as predefined strain localization. It must be mentioned, however, that due to the initial strain concentration for this type of specimen a local strain measurement is obligatory. Moreover, while the local strain measurement can be focused on this area for this specimen, the whole specimen surface must be measured for ISO 527.

Due to the different types of strain localization the stress-strain response of ISO18872 specimens at high loading rates and high strains is not comparable to that of ISO527 specimens without taking other effects into account. This makes it difficult to compare these tests with other uniaxial tensile tests.

Furthermore, ISO 18872 requires either a new injection moulding tool or the smooth notch should be machined from the injection moulded specimens. Due to the remove of injection moulded skin layer the injection moulded and machined specimen would not provide the same results.

The material models in FE codes require true stress-strain data under constant strain rate. However, due to the inevitable necking of bulk polymeric tensile specimens, the determination of true-stress strain curves under constant strain rate is a challenging task. Global displacement controlled tests results always in increasing strain rate and hence does not fulfil the model requirements. The future experimental work will focus on the design of local strain controlled tensile tests and the comparison of these results with test without strain localization (i.e., confined compression). 


\section{References}

1. ISO 527-2:1993, Plastic Test Standard (1993)

2. ISO 18872:2007, Plastic Test Standard (2007)

3. E. Parsons, M.C. Boyce, D.M. Parks, Polymer, 45, 2665-2684 (2004)

4. E.M. Parsons, M.C. Boyce, D.M. Parks M. Weinberg, Polymer, 46, 2257-2265 (2005)

5. X. Xiao, Polymer Testing, 27, 164-178 (2008)

6. G. Dean, B. Read, Polymer Testing, 20, 677-683 (2001)

7. C. G'Sell, S.L. Bai, J.M. Hiver, Polymer, 45, 5785-5792 (2004)

\section{Acknowledgments}

Parts of this project were performed at the Polymer Competence Center Leoben $\mathrm{GmbH}$ within the $\mathbf{K}_{\text {plus }}{ }^{-}$ programme of the Austrian Ministry of Traffic, Innovation and Technology. The funding within this programme by the Governments of Austria, Styria and Upper Austria is gratefully acknowledged. 